\title{
Keeping an eye on gold
}

\author{
Olivier Pluchery • Hynd Remita • Pierre-François Brevet • \\ Stéphane Roux
}

Published online: 18 December 2013

(C) The Author(s) 2013. This article is published with open access at SpringerLink.com

Unlike the metal itself, the gold markets are always changing and you have to have your eyes wide open to keep up. This is especially true in the field of gold nanotechnology, where scientists around the world are advancing our understanding of this special form of gold year in, year out.

This special issue of Gold Bulletin offers a series of 16 research articles describing cutting edge advances in the field of gold nanotechnology. The common thread of this work is the GDR Or-nano, a research network set up in France in 2006 with the support of CNRS (Centre National de la Recherche Scientifique, the French fundamental research organization). Or-nano (which stands for gold-nano) has organized and run a wide variety of meetings and events over the last 8 years, involving approximately 450 researchers along the way.

The successes of Or-nano community are numerous and include a variety of new research collaborations, the launch of

O. Pluchery $(\bowtie)$

Université Pierre et Marie Curie (UPMC),

4 place Jussieu, 75005 Paris, France

e-mail: olivier.pluchery@insp.jussieu.fr

\section{H. Remita}

Université Paris-Sud, 91405 Orsay Cedex, France

\section{P.-F. Brevet}

Université Claude Bernard Lyon-1,

43 Boulevard du 11 Novembre 1918, 69622 Villeurbanne cedex, France

\section{S. Roux}

Université de Franche-Comté,

16 route de Gray, 25030 Besançon cedex, France
Keeping an eye on gold.

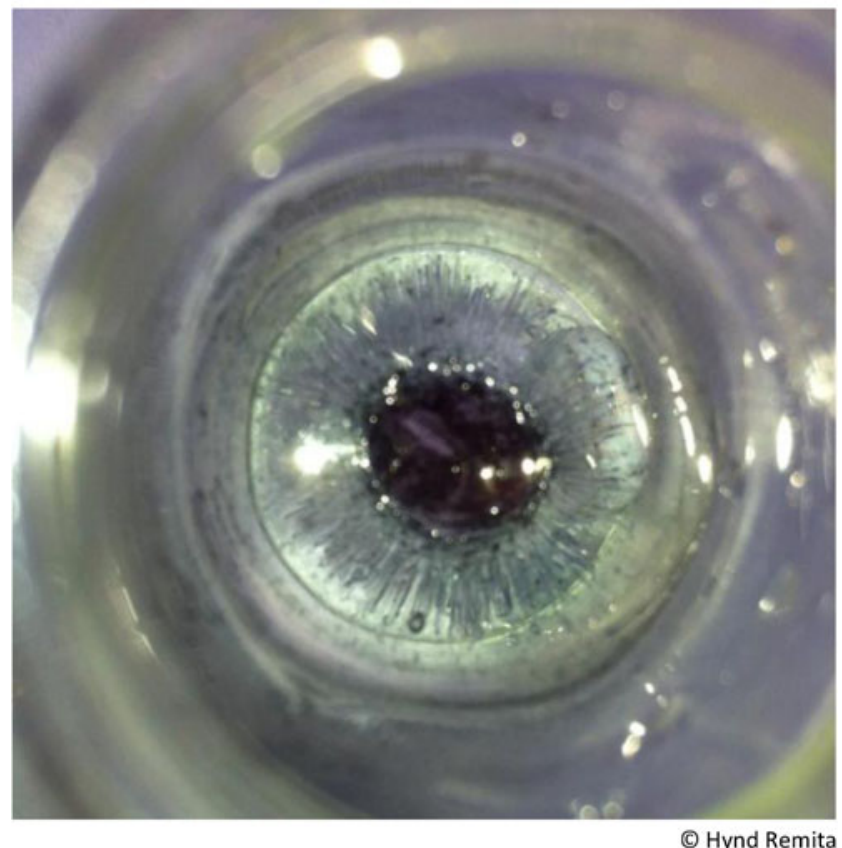

Suspension of gold nanoparticles stabilized by citrate in frozen water. The photo is taken from above, with the beaker edge out of focus in the foreground.

new projects, many invited lectures, and the training of the next generation of $\mathrm{PhD}$ students and postdocs.

In early 2013, a call to the community was issued for submissions to Gold Bulletin. After the usual review process, 16 articles have been accepted, bringing together 86 authors and 17 different research institutions into this special issue. This is a fairly accurate snapshot of the work achieved by Or- 
nano during its activity. Indeed, one third of the manuscripts describe research completed as a result of new collaborations setup through Or-nano. The scientific scope of this issue is vast, spanning many different topics such as plasmonics, catalysis, electrocatalysis, medical therapeutics, chemical synthesis, assemblies on surfaces, coloration of glass, and even education.

Finally, we dedicate this collection of articles to Professor Catherine Louis for her tireless work as a coordinator of Ornano over the last 8 years, where she has maintained the pace of the network admirably.

The page will now be turned, and Or-nano will become gold-nano seeking to replicate its success in France to Europe and beyond. We will be keeping our eyes open and looking forward to continued progress in the field of gold nanotechnology

Olivier Pluchery, Hynd Remita, Pierre-François Brevet, and Stéphane Roux

Guest editors for this Gold Bulletin issue

Open Access This article is distributed under the terms of the Creative Commons Attribution License which permits any use, distribution, and reproduction in any medium, provided the original author(s) and the source are credited. 